\title{
Correction to: Evaluation of Cardiovascular Disease Risk in HIV-1-Infected Patients Treated with Darunavir
}

Magda Opsomer $^{1}$ - Dessislava Dimitrova ${ }^{2} \cdot$ Johan Verspeelt $^{1} \cdot$ Amy Purrington $^{3} \cdot$ Abdul Mehbob $^{4} \cdot$ Scott Chavers $^{2}$. Helen $\mathrm{Pai}^{5} \cdot$ Simon Vanveggel ${ }^{1} \cdot$ Donghan Luo $^{2} \cdot$ Kimberley Brown $^{6} \cdot$ Christiane Moecklinghoff $^{7} \cdot$ Richard E. Nettles $^{6}$. Katia Boven ${ }^{2}$

Published online: 6 August 2018

(c) The Author(s) 2018

\section{Correction to: Drugs in R\&D \\ https://doi.org/10.1007/s40268-018-0238-8}

In the original publication of the article, Table 2 has been published incorrectly. The corrected Table 2 is shown below:

The original article can be found online at https://doi.org/10.1007/ s40268-018-0238-8.

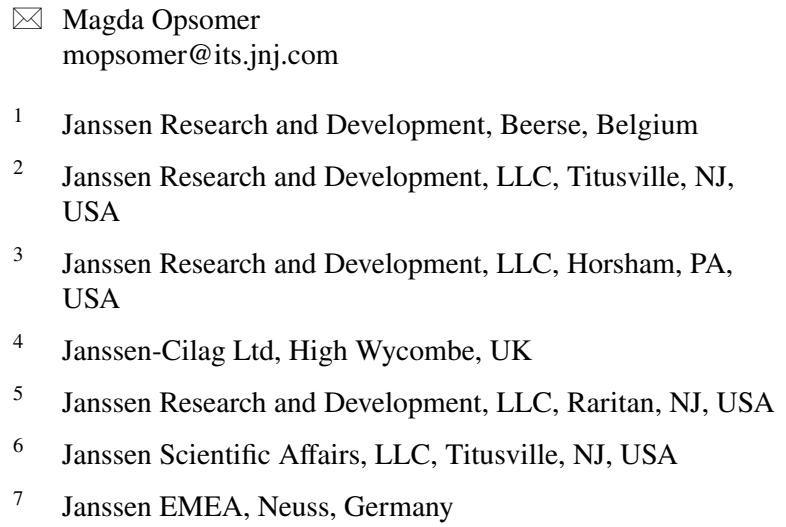


Table 2 Signal scores from FAERS (2016Q2): cardiovascular and cerebrovascular events

\begin{tabular}{|c|c|c|c|c|c|c|c|c|c|c|c|c|}
\hline \multirow[t]{3}{*}{ Event* } & \multicolumn{12}{|c|}{ First-generation PIs } \\
\hline & \multicolumn{3}{|c|}{ Indinavir } & \multicolumn{3}{|c|}{ Nelfinavir } & \multicolumn{3}{|c|}{ Ritonavir } & \multicolumn{3}{|c|}{ Saquinavir } \\
\hline & $n$ & EBGM & EB05 & $n$ & EBGM & EB05 & $n$ & EBGM & EB05 & $n$ & EBGM & EB05 \\
\hline Carotid artery occlusion & 1 & 0.966 & 0.213 & NR & NR & NR & 3 & 1.057 & 0.405 & 1 & 1.021 & 0.225 \\
\hline Carotid artery stenosis & 9 & 3.523 & 2.003 & 2 & 1.097 & 0.347 & 4 & 1.008 & 0.436 & 1 & 0.765 & 0.169 \\
\hline Carotid artery thrombosis & 2 & 1.535 & 0.486 & NR & NR & NR & NR & NR & NR & NR & NR & NR \\
\hline Coronary artery insufficiency & 1 & 1.23 & 0.271 & NR & NR & NR & NR & NR & NR & NR & NR & NR \\
\hline Coronary artery occlusion & 32 & 3.275 & 2.432 & 10 & 1.517 & 0.889 & 35 & 1.975 & 1.486 & 16 & 3.147 & 2.063 \\
\hline Coronary artery thrombosis & 2 & 1.367 & 0.433 & 1 & 0.636 & 0.14 & 2 & 0.635 & 0.201 & 4 & 2.846 & 1.227 \\
\hline Coronary artery stenosis & 5 & 1.959 & 0.922 & 11 & 4.151 & 2.488 & 16 & 2.405 & 1.576 & 4 & 1.998 & 0.864 \\
\hline Ischemic stroke & 1 & 0.505 & 0.111 & NR & NR & NR & 6 & 0.627 & 0.315 & NR & NR & NR \\
\hline Sudden cardiac death & NR & NR & NR & NR & NR & NR & 2 & 0.494 & 0.157 & 1 & 0.915 & 0.202 \\
\hline Ischemic heart disease (SMQ) & 373 & 1.802 & 1.653 & 253 & 1.85 & 1.667 & 494 & 1.16 & 1.077 & 177 & 1.896 & 1.673 \\
\hline Myocardial infarction (SMQ) & 325 & 1.912 & 1.744 & 229 & 2.016 & 1.806 & 430 & 1.22 & 1.126 & 161 & 2.08 & 1.824 \\
\hline $\begin{array}{l}\text { CNS hemorrhages and cerebro- } \\
\text { vascular conditions (SMQ) }\end{array}$ & 154 & 0.911 & 0.796 & 75 & 0.594 & 0.49 & 297 & 0.78 & 0.708 & 70 & 0.876 & 0.717 \\
\hline \multirow[t]{3}{*}{ Event* } & \multicolumn{12}{|c|}{ Second-generation PIs } \\
\hline & \multicolumn{3}{|c|}{ Atazanavir } & \multicolumn{3}{|c|}{ Darunavir } & \multicolumn{3}{|c|}{ Fosamprenavir } & \multicolumn{3}{|c|}{ Tipranavir } \\
\hline & $n$ & EBGM & EB05 & $n$ & EBGM & EB05 & $n$ & EBGM & EB05 & $n$ & EBGM & EB05 \\
\hline Carotid artery occlusion & NR & NR & NR & NR & NR & NR & 1 & 1.082 & 0.238 & NR & NR & NR \\
\hline Carotid artery stenosis & NR & NR & NR & NR & NR & NR & NR & NR & NR & 3 & 2.829 & 1.063 \\
\hline Carotid artery thrombosis & NR & NR & NR & NR & NR & NR & NR & NR & NR & NR & NR & NR \\
\hline Coronary artery insufficiency & NR & NR & NR & NR & NR & NR & NR & NR & NR & NR & NR & NR \\
\hline Coronary artery occlusion & 5 & 0.577 & 0.272 & 6 & 1.192 & 0.598 & 2 & 0.86 & 0.272 & 1 & 0.75 & 0.165 \\
\hline Coronary artery thrombosis & 5 & 1.914 & 0.901 & NR & NR & NR & NR & NR & NR & NR & NR & NR \\
\hline Coronary artery stenosis & 10 & 2.189 & 1.282 & 4 & 1.732 & 0.749 & 3 & 1.761 & 0.675 & NR & NR & NR \\
\hline Ischemic stroke & NR & NR & NR & 3 & 0.744 & 0.285 & NR & NR & NR & NR & NR & NR \\
\hline Sudden cardiac death & 6 & 1.919 & 0.963 & 2 & 0.912 & 0.289 & NR & NR & NR & NR & NR & NR \\
\hline Ischemic heart disease (SMQ) & 176 & 0.881 & 0.777 & 140 & 1.112 & 0.966 & 37 & 0.884 & 0.671 & 8 & 0.382 & 0.21 \\
\hline Myocardial infarction (SMQ) & 149 & 0.894 & 0.78 & 121 & 1.169 & 1.005 & 33 & 0.929 & 0.693 & 6 & 0.335 & 0.168 \\
\hline $\begin{array}{l}\text { CNS hemorrhages and cerebro- } \\
\text { vascular conditions (SMQ) }\end{array}$ & 102 & 0.565 & 0.479 & 93 & 0.793 & 0.667 & 24 & 0.679 & 0.482 & 29 & 1.585 & 1.159 \\
\hline
\end{tabular}

The threshold for disproportional reporting was $n \geq 3, \mathrm{EBGM} \geq 2$, and EB05 $>1$. Events that met the disproportionality threshold are in bold 2016Q2 2016 quarter 2, CI confidence interval, CNS central nervous system, FAERS US Food and Drug Administration Adverse Event Reporting System, EBGM Empirical Bayesian Geometric Mean, EB05 lower bound of the 2-sided 90\% CI around EBGM, MedDRA Medical Dictionary for Regulatory Activities, NR not reported, PIs protease inhibitors, SMQ Standardized MedDRA Query

*Events based on MedDRA preferred terms or SMQs (as noted)

Open Access This article is distributed under the terms of the Creative Commons Attribution-NonCommercial 4.0 International License (http://creativecommons.org/licenses/by-nc/4.0/), which permits any noncommercial use, distribution, and reproduction in any medium, provided you give appropriate credit to the original author(s) and the source, provide a link to the Creative Commons license, and indicate if changes were made. 